\title{
Téoros
}

Revue de recherche en tourisme

\section{Une expérience différente : les Iles-de-la-Madeleine}

\section{Yvonne Langford}

Volume 13, numéro 1, mars 1994

Bas-Saint-Laurent, Gaspésie, Iles-de-la-Madeleine : fascinantes périphéries

URI : https://id.erudit.org/iderudit/1077770ar

DOI : https://doi.org/10.7202/1077770ar

Aller au sommaire du numéro

Éditeur(s)

Université du Québec à Montréal

ISSN

0712-8657 (imprimé)

1923-2705 (numérique)

Découvrir la revue

Citer cet article

Langford, Y. (1994). Une expérience différente : les Iles-de-la-Madeleine.

Téoros, 13(1), 19-21. https://doi.org/10.7202/1077770ar d'utilisation que vous pouvez consulter en ligne.

https://apropos.erudit.org/fr/usagers/politique-dutilisation/ 


\section{Une expérience différente: les Iles-de-la-Madeleine}

\section{Yvonne Langford"}

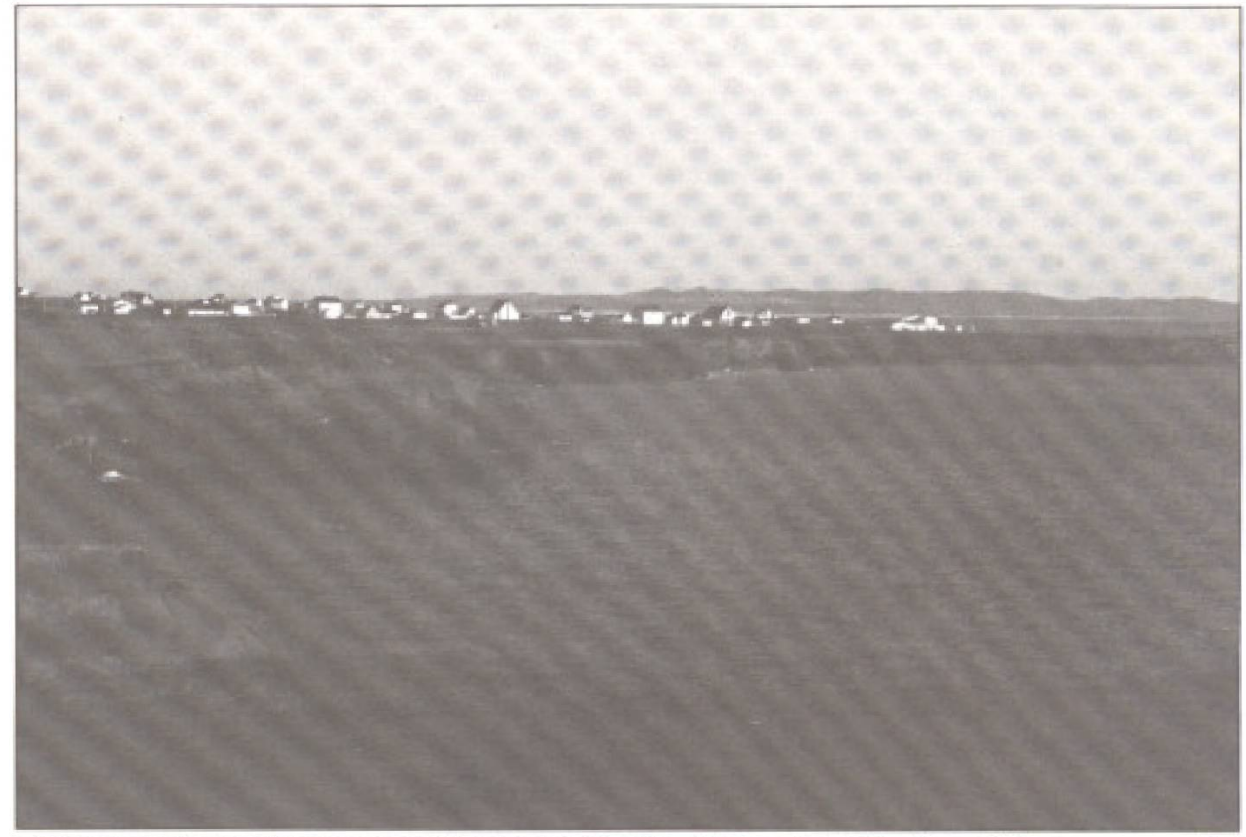

L'intérêt touristique des lles tient d'abord à l'originalité, à la beauté et à la diversité des paysages et des éléments culturels. Photo : Marc Laplante.

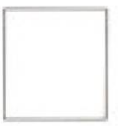

Les Iles-de-la-Madeleine constituent sans contredit la région du Québec où le visiteur étranger à l'archipel ressentira le dépaysement dans son expression la plus complète; fut-ce sur le plan culturel ou sur celui du milieu naturel, les Iles présentent un visage qui ne ressemble en rien à ce qu'on connaît ailleurs au Québec:

L'archipel des Iles-de-la-Madeleine correspond en effet, à s'y méprendre, à ce que nos rêvves les plus secrets imagineraient être une île véritable, lointaine, isolée, perdue dans la mer. Les Iles-de-la-Madeleine sont néanmoins habitées et ce, de façon assez dense; elles font également partie intégrante du territoire québécois, ce qui n'enlève rien, loin de là, à leur charme exotique. ${ }^{(2)}$

Depuis une vingtaine d'années, les Québécois découvrent les Iles-de-laMadeleine. Ils paraissent séduits par l'originalité des lieux et de ses habitants. Ceux qui les connaissent y viennent régulière- ment et leurs commentaires enthousiastes finissent souvent par convaincre leurs proches d'yvenir envacances. Les autres neles connaissent pas encore, mais ne les ignorent pas pour autant, ceux-là entretenant un projet de voyages aux Iles «comme une traite à se payer au moins une fois dans sa vies ${ }^{(3)}$.

Cet engouement a fait en sorte, depuis les années 1970, de développer aux lles une industrie touristique qui compte aujourd'hui pour la deuxième activité économique en importance, après la pêche. Cernons donc un peu mieux cette réalité du tourisme aux Iles-de-la-Madeleine et voyons de quelle façon les gens des Iles envisagent le futur de ce secteur en croissance.

\section{Un développement récent et rapide}

*Rares étaient ceux qui, il y a une vingtaine d'années, osaient prévoir la vocation touristique des Iles-de-la-Madeleine, à tout le moins son ampleur, $\$^{(4)}$ La mise en service
Madame Yvonne Langford est professeure av Cegep de la Gaspósie et des lles. 
d'un traversier entre les Iles et l'Ile du Prince-Edouard en 1971 a tout à coup rendu l'archipel plus accessible. C'est à partir de ce moment-là que les vacanciers sont venus, de plus en plus nombreux, visiter l'archipel jusqu'alors presqu'ignoré des Québécois. La très forte croissance de la fréquentation touristique, liée à un manque d'organisation et d'infrastructures, fera en sorte qu'en dépit de leur accueil légendaire, les Madelinots manifesteront, vers la fin des années 1970 , une certaine inquiétude devant l'ampleur et le caractère soudain du phénomène. De cette époque datent les rumeurs, souvent exagérées, d'envahissement, de dérangement... Les efforts d'organisation et l'implantation d'infrastructures d'accueil plus adéquates viendront atténuer, puis éteindre ces réactions que le flot subit de visiteurs avait d'abord suscitées dans la vie tranquille des Madelinots. \&L'activité touristique n'a plus le caractè̀e menaçant et envahissant qu'elle pouvait avoir, au début des années 1970, aux yeux de l'ensemble de la population. On s'y est en quelque sorte apprivoisé, on se l'est approprié et on a ajusté progressivement les infrastructures aux besoins (...) w $w^{(9)}$ Depuis, le tourisme fait pleinement partie de la viécoonomiquedes Iles. Il est aussi un élément de la dynamiquedu milieu, avecce que cella peut signifier sur le plan socio-culturel.

\section{Une destination originale}

L'intếrêt touristique des lles tient d'abord a l'originalité, à la beauté et à la diversité des paysages(mer, plages, dunes, fal aises, faune et flore, caractèreinsulaire) et auxéléments culturels (architecture, histoire, culture matếrielle, particularitésculturelles, cuisine régionale, etc.). Les paysages des Iles constituent done la matière première de l'industrie touristique régionale. Ils composent un tout original et varié enrichi par la culture madelinienne héritée à la fois de l'Acadie et du Québec.

Depuis quelques années, les Iles ont aussi à peu près tout ce qu'il faut comme infrastructures d'accueil et de support. L'archipel est relié quotidiennement au continent par voie aérienne (trois compagnies offraient sept vols quotidiens à l'été 1993) et maritime(letraversier effectuedeux liaisons quotidiennes avec I'Tle du Prince-Edouard en haute saison). L'offre hôtelière se raffine d'anncée en année et est complétée par quelques campings et un réseau d'hébergement chez les Madelinots (unique au Québec). Basée sur les ressources de la mer, la restauration s'est gagnée une réputation enviable. Les artistes et artisans présentent des produits originaux et des spectacles en tous genres. Les activités offertes aux visiteurs sont nombreuses et variées: musée, aquarium, site historique, plongée sous-marine, équitation, pêche, voile, planche à voile, thalassothérapie, concours de châteaux de sable, fẹtes acadiennes, etc.

Les visiteurs prennent plaisir à vivre dans un contexte insulaireet maritime, aurythme des habitants, partageant avec eux, le temps d'une semaine ou deux, ce que sont les Iles.

\section{Une clientèle fidèle, satisfaite et en croissance}

Les visiteurs des Iles-de-la-Madeleine se disent très satisfaits de leur séjour. $\mathrm{Par}$ exemple, une étude réalisée à l'été 1993 révèle que $98,1 \%$ des visiteurs recommanderont un voyage de vacances aux Iles à leurs parents et amis et $94 \%$ estimaient que leur perception du milieu a correspondu également ou en mieux à l'idée qu'ils en avaient avant de le connaître. Voilà ce qui constitue sûrement le meilleur instrument de promotion touristique: un bouche-àoreille positif.

Associée aux moyens promotionnels mis en oeuvre par l'Association touristique des Iles, cette réputation grandissante s'est d'ailleurs soldée par une forte croissance de fréquentation touristique depuis 1984 ( 66 $\%$ d'augmentation entre 1984 et 1991). Le nombre de visiteurs estivaux a atteint le chiffre record de 30590 à l'été 1991 . Ces résultats impressionnants ont cependant été assombris par une baisse cumulée de quelque $15 \%$ pour 1992 et 1993 , quoique les facteursconsidéréscommeresponsables de cette diminution sont plutôt conjoncturels que structurels: hausse des tarifs du transport aérien, mauvaise température en début d'été, menace de grève sur le traversier, etc.

Il ne faut cependant pas en conclure que les Iles s'enfoncent sous le poids des trop nombreux touristes. Les hausses de clientèles se sont surtout faitsentir pendant les mois de juin et d'août.

Les études rélisées par l'Association touristique des Iles indiquent aussi une grande dispersion du profil socio-économique de la clientèle, avec une tendance vers les classes les plus favorisées de la société́, cette observation va de pair avec le coût somme toute assez élevé du produit touristique madelinien. Les vacanciers sont principalement originaires de Montréal et de Québec $(53 \%)$. $65 \%$ en étaient à leur première visite dans l'archipel à l'été 1993. Enfin, la durée moyenne de séjour est de 7,92 nuitées; les lles ressortent clairement comme une destination de long séjour.

\section{Des forces, mais aussi des contraintes}

Voilà un portrait bien positif de la situation. Mais le tourisme madelinien a aussi ses problèmes et ses défis. Allons-y de quelques exemples. Disons d'abord que le point fort des lles, le milieu naturel et culturel, reste fragile: les dunes sont *squattérisées» et envahies par des véhicules motorisés, les réserves d'eau douce sont menacées, de belles buttes sont déchiquetées par les bulldozers, l'architecture se banalise, le mode de vie se modernise, etc. La durée réduitedela saison estivale amène, en corollaire, une sous-utilisation du potentiel et des infrastructures touristiques, ce qui fait que bien des entreprises touristiques connaissent la précarité. Autre défi de taille: les lles sont loin des grands centres de population, donc de leurs marchés.

\section{Quelle approche privilégier: développement durable ou rentabilité immédiate maximale?}

Réalisé en 1988, le plan de développernent touristique a permis de dresser un portrait précis de la situation, puis d'établir les priorités d'intervention autour desquelles s'articulent depuis lors les efforts de développement touristique:

- prioritairement, étaler la saison;

- maintenir une bonne image auprès d'une clientèle fidèle et de qualité;

- ne pas ajouter à la détérioration du milieu et, autant que possible, le préserver;

- diversifier les clientèles et rejoindre des micro-segments spécialisés.

Les gens des Iles ont été unanimes à reconnaître le bien-fondé de l'approche globale prônée par le plan de développe- 
ment. C'est donc dans ce sens qu'ils se sont efforcés, ces dernières années, d'améliorer et de diversifier le produit touristique, tout en étant convaincus que le tourisme devait chercher à s'intégrer le plus entièrement possible à la dynamique des Iles. Ilss'appliquent à mieux respecter les conditions propres au développement touristique via le schéma d'aménagement et les plans d'urbanisme. Ils amorcent la mise en forme de sous-produits susceptibles d'attirer les visiteurs en basse saison (par exemple, l'observation des phoques etdes blanchons en mars, ou la tenue de petits congrès et de réunions en mai, juin, septembre et octobre). Ils améliorentcertaines infrastructures et diversifient les activités offertes aux visiteurs. Sur ce plan du développement touristique, il reste encore à faire, mais la concertation bien amorcée des acteurs touristiques, économiques et municipaux est un actif.

Parallèlement, l'Association touristique a misl'emphase sur le marketing du produit. La stratégievisaità ren forcer la notoriété ct le positionnement des Iles sur les marchés existants, à diversifier les clientèles par l'ouverture de nouveaux marchés et à poursuivre certaines démarches auprès de clientèles-cibles en fonction de nouveaux produits touristiques axés sur la basse saison. Conjugués aux efforts d'amélioration et de diversification du produit touristique, ces investissements connaissent des applications qui débouchent sur des résultats de plus en plus tangibles.

Globalement, la situation est positive. Les marchés offrent des potentiels réalistes de développement. L'accentuation des efforts de positionnement porte fruit. Malgré la multiplicitédes intervenants, les acteurs du développement touristique démontrent une concertation qu'il est nécessaire d'accentuer, mais qui est déjà efficace. Enfin, quoique nécessitant une certaine mise à jour, le plan de développement touristique a permis de disposer d'un état de situation et d'orientations précises en matière de développementetdemarketing auxquelles le milieu dans son ensemble a adhéré et qui inspirent encore largement les initiatives régionales.

Par contre, ce constat positif ne doit pas faire oublier que le tourisme est une industrie très vulnérable - les pêcheurs et travailleurs de la mer savent eux aussi maintenant que la pêche est beaucoup plus fragile qu'il n'y paraissait - et sensible à de nombreux facteurs dontune partie échappe à tout contrôle des Madelinots. De plus, des menaces pèsent sur le produit touristique et des efforts sont nécessaires pour le parfaire. Par ailleurs, la mise en valeur de la basse saison représente un défi de taille. La petitesse des entreprises touristiques madeliniennes est une autre réalité avec laquelle il faudra composer. Et, à l'échelle mondiale, le tourisme devra affronter des défis et composer avec de nouvelles tendances susceptibles d'avoir des impacts aux Iles comme ailleurs; qu'on pense par exemple au fractionnement des vacances, à l'évolution socio-démographique de la population, au déplacement des marchés, à l'agressivité croissante de la concurrence ou aux exigences croissantes des consommateurs.

Les résultats obtenus de la mise en oeuvre du plan de développement touristique ont fait la preuve qu'il était possible de privilégier une double stratégie de développement, c'est-à-dire de viser la croissance des retombées touristiques tout en misant sur uneindustrie touristiquesignificativeà long terme pour l'ensemble de la communauté. Cette approche est la seule valable dans les circonstances. Espérons que les résultats mitigés des deuxdernières saisons estivales ne viendront pas remettre en cause cette vision originale et cette façon de faire du développement touristique qui a été celle des lles au cours des dernières années. $f$

\section{NOTES}

(1) Pierre Rastoul et Gilles Rousseau, Les lles-de-laMadeleine, Itinúr aire culturel, Montréal, France-Amérique/Éditeur officiel du Cuébec, 1979. p. 9

(2) Itbid. p. 12.

(3) Madeleine Dubuc, Le Guide du Oubbec, Montréal, Editions La Presse, 1983, p. 261.

(4) MRC des lles Ueannot Gagnon, rédacteur), Schéma d'aménagement de la MRC dess llesdo-la-Madeleine, Cap-aux-Meules, s.é, 1987, p. 18.

(5) bid., p. 19 